\title{
Training Neural Network as Approximate 4:2 Compressor applying Machine Learning Algorithms for Accuracy Comparison
}

\author{
Lavanya Maddisetti ${ }^{1}$, Ranjan K. Senapati ${ }^{2}$, JVR Ravindra ${ }^{3}$ \\ ${ }^{1}$ Research Scholar, Department of ECE, KL Deemed to be University, lavanya.maddisetty@ gmail.com \\ ${ }^{1}$ Assistant Professor, Department of ECE, C-ACRL, Vardhaman College of Engineering, India \\ ${ }^{2}$ Professor, Department of ECE, KL Deemed to be University, rksphd@ gmail.com \\ ${ }^{3}$ Professor, Department of ECE, C-ACRL, Vardhaman College of Engineering, India, jayanti@ieee.org
}

\begin{abstract}
This research work is on interlinking the emerging application namely machine learning algorithm and an approximation technique to compare the accuracy and loss values of the trained machine learning based approximate $4: 2$ compressor model in accordance with the proposed approximate 4:2 compressor such that the trained model is sufficient to observe the performance of the approximate model without performing error analysis separately using MATLAB. The key attention of this work is to adopt truth tables from an exact and proposed 4:2 compressor as data set and train the artificial neural network as the respective 4:2 compressor model. The Neural Networks are trained for two data sets, firstly by applying inputs and only Sum output and second with inputs and three $\left(\mathrm{C}_{\text {out }}\right.$, Carry and Sum) outputs. Error Analysis of approximate 4:2 compressor has been performed with MATLAB and training of artificial neural network has been done employing Anaconda Python with Jupyter Integrated Development Environment. A comparison of error rate has been made for exact and approximate 4:2 compressors and accuracy has been evaluated for trained neural networks (as exact and approximate 4:2 compressors) and found that the loss values are smaller and the difference between the trained accuracy values are less for trained and validated proposed approximate machine learning model.
\end{abstract}

Key words: Approximate Circuits, Compressor, Neural Network, Supervised Learning

\section{INTRODUCTION}

Addition and multiplication operations are the basic computations that occur in any processors. While low power and high speeds are the important prerequisites for all the portable devices and processing units. To increase the speed and reduce the power consumption adders in the multipliers should be replaced by an element which consumes less area and power. Hence, compressors [1-2] have been employed instead of ripple carry adders in the partial product reduction stage of a multiplier. For additional reduction in power, approximation techniques have been used in the literature. Among many approximation techniques, probabilistic pruning type of approximation method has been employed in this research work. These compressors with approximation methods can be applied to Machine Learning (ML) algorithms to train the Artificial Neural Network (ANN) as the ML based compressor models. The main intention to apply ML algorithms is to observe how the trained Neural Network (NN) is handling accuracy for the applied datasets of exact and approximate compressors. To train the ANN as the approximate 4:2 compressor models, supervised classification machine learning algorithm has been utilized and the performance of the trained ML model has been determined by observing the accuracy and loss values of the train set and test set accuracy is measured from the confusion matrix of the machine learning model.

The forthcoming sections of the paper are organized as follows. Section 1 gives the previous research of compressors, approximate circuits, and machine learning. The proposed approximate 4:2 compressor and its corresponding trained $\mathrm{NN}$ is shown in section 3. Simulation results in terms of error metrics and accuracy, loss values are given for 4:2 compressors and ML based compressors respectively in section 4 and the research work has been concluded with section 5 .

\section{RELATED WORK}

The authors of [1] have proposed 3:2, 4:2 and 5:2 compressors with XOR-XNOR and \$2ltimes 1 \% multipliexers to achieve low power and high speed compressor architectures unlike XOR based compressors. Various XOR-XNOR circuits with different number of transistors have been presented in paper [2] to place them in 4:2 and 5:2 compressors. In [3], the authors have made the comparison of three 4:2 compressors where they vary by XOR gates and CMOS+ MUX gates to reduce the power consumption and increase the speed. Approximations have been implemented on 4:2 compressors in [4-5] and proposed two 4:2 compressor designs and have been replaced them in multiplier to observe the performance of the multiplier with the proposed compressors and concluded finally with image processing application where approximations do not influence it to a large extent. The authors of [6] have proposed an approximation technique named probabilistic pruning where a node or interconnection or any block of a circuit is deleted to reduce power consumption and area of any architecture. When approximations have been implemented on any circuit 
then there will be variation in the exact and approximate outputs and the difference in these outputs can be calculated from error distance, mean error distance and normalized error distances which were presented in [7-8]. Machine Learning can be applied to VLSI circuits interrelating ML and CAD algorithms. In [9-10], the authors have been reviewed existing CAD algorithms which can take advantage from ML and summarized the important challenges with different approaches. In [11], the author explained that the chip design process is common for all the applications. Hence, if this data process is made common then chip designing can be made automated. The majority voting method is developed in [12].

\section{PROPOSED WORK}

This research work has been divided into two sections where the first section illustrates the way approximation technique has been applied to exact 4:2 compressor and the second part demonstrates the training of ANN as exact and approximate 4:2 compressors to find accuracy of the trained ML models. The performance of an approximate compressor can be observed by calculating error rate and no. of errors with respect to the other inexact compressors and the performance of the ML model can be computed from the accuracy and loss values of the trained and validated models. With these two sections, the accuracy comparison of approximated compressor and trained ML model has been done and evaluated whether or not the trained ML models are giving accuracies according to the error metrics of the exact and approximate 4:2 compressors (Accuracy of exact and approximate 4:2 compressors are shown with respect to the error rate and no. of errors).

\subsection{Approximate 4:2 Compressor}

The approximate technique employed on the exact 4:2 compressor is probabilistic pruning [6] where XNOR1 and XNOR2 terminals in the XOR-XNOR1 and XOR-XNOR2 circuits of an exact compressor [1] has been removed respectively. With this, the proposed approximate 4:2 compressor has been shown in Figure 1.

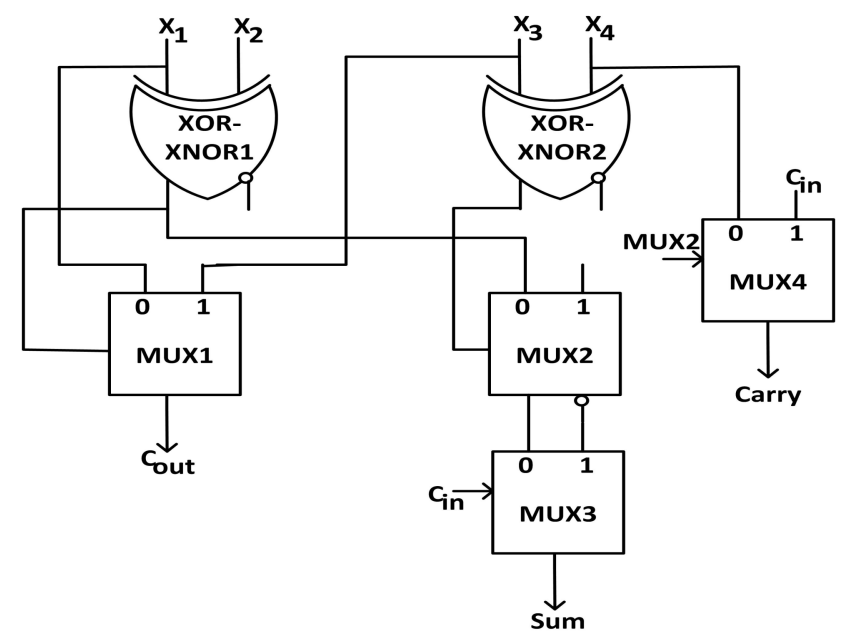

Figure 1: Proposed Approximate 4:2 Compressor
For Figure 1, the Sum and Carry expressions of the proposed 4:2 compressor are given in eq. (1) and eq. (2)

$$
\begin{gathered}
\text { Sum }=C_{\text {in }}\left[\left(X_{1} \oplus X_{2}\right)\left(X_{3} \oplus X_{4}\right)^{\prime}\right]^{\prime}+ \\
C_{\text {in }}^{\prime}\left[\left(X_{1} \oplus X_{2}\right)\left(X_{3} \oplus X_{4}\right)^{\prime}\right] \\
\text { Carry }=X_{4}\left[\left(X_{1} \oplus X_{2}\right)\left(X_{3} \oplus X_{4}\right)^{\prime}\right]^{\prime}+ \\
C_{\text {in }}\left[\left(X_{1} \oplus X_{2}\right)\left(X_{3} \oplus X_{4}\right)^{\prime}\right]
\end{gathered}
$$

Removing XNOR2 terminal does not change any of the outputs of 4:2 compressor since it is not connected to any of the terminal or node as observed from [1]. But with the deletion of XNOR1 terminal the $\mathrm{C}_{\text {out }}$, Carry and Sum outputs are varying by 0,12 and 10 respectively when compared with the exact $4: 2$ compressor. When the accuracy is compared with [4], the respective errors are found to be 6,8 and 12 for $\mathrm{C}_{\text {out }}$, Carry and Sum. Therefore, the proposed inexact 4:2 compressor is more accurate than [4] as the total number of errors and error rates are less.

\subsection{Neural Network as Approximate 4:2 Compressor}

Supervised classification type of machine learning algorithm has been utilized to train the neural network as the approximate 4:2 compressor. The purpose of employing classification ML algorithm is to analyze the outputs as ' 1 ' and ' 0 ' from the obtained true and false values of the predicted test set. This is because if the train and test set accuracies are equal then we can make declaration that the trained ML model is acting in accordance with the exact or proposed 4:2 compressor. The truth table of pruned approximate 4:2 compressor has been given as input to the neural network to find the accuracy of the train set and predict accuracy of the unseen test set. This gives the performance of the machine learning model functioning as proposed approximate compressor. The artificial neural network with truth table as data set is displayed in Figure 2, the data set shown here is with the three outputs.

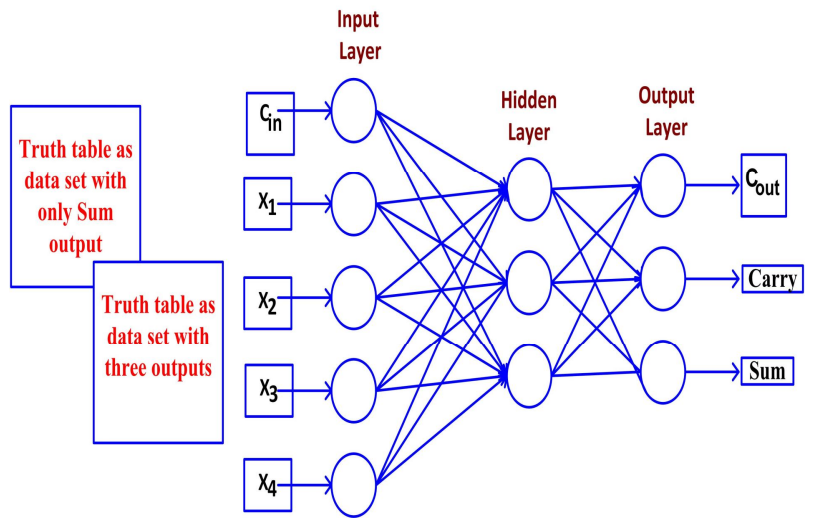

Figure 2: Artificial Neural Network with Truth tables as Datasets 
The ML based approximate 4:2 compressor becomes a complete validated model when the

- Loss values are smaller.

- Difference in trained (before K4 cross validation) and validated (after K4 cross validation) accuracy values are less.

- Mean value is high and variances between the trained and validated accuracy values are less to obtain low bias and low variance ML model.

\section{EXPERIMENTAL RESULTS}

The simulation of approximate 4:2 compressor for supervised machine learning algorithm has been implemented employing Anaconda python and Jupyter Integrated Development environment. The proposed approximate 4:2 compressor is taken for which error parameters have been calculated using MATLAB. The error analysis of approximate compressor has been performed using error metrics of [7] and to check the performance of the trained machine learning model as approximate 4:2 compressor, the accuracy has been computed by taking only Sum output first and then taking $\mathrm{C}_{\text {out }}$, Carry and Sum outputs. When probabilistic pruning type of approximation technique has been implemented on exact 4:2 compressor, there is difference in exact and approximate outputs for $\mathrm{C}_{\text {out }}$, Carry and Sum. Table 1 shows the number of errors and error rate for each of these outputs of 4:2 compressors.

Table 1: Error Analysis of 4:2 Compressors

\begin{tabular}{|c|c|c|c|c|c|c|}
\hline \multirow{2}{*}{$\begin{array}{c}4: 2 \\
\text { Compressors }\end{array}$} & \multicolumn{3}{|c|}{ Errors } & \multicolumn{3}{c|}{ Error Rate } \\
\cline { 2 - 7 } & $\mathbf{C}_{\text {out }}$ & $\begin{array}{c}\text { Carr } \\
\mathbf{y}\end{array}$ & Sum & $\mathbf{C}_{\text {out }}$ & $\begin{array}{c}\text { Carr } \\
\mathbf{y}\end{array}$ & Sum \\
\hline Exact [1-2] & 0 & 0 & 0 & 0 & 0 & 0 \\
\hline Approx.[4] & 6 & 8 & 12 & 18.75 & 25 & 37.5 \\
\hline Proposed & 0 & 12 & 10 & 0 & 37.5 & 31.25 \\
\hline
\end{tabular}

\subsection{Simulations of Trained ML models}

In order to train the neural network, the split data is sent as input and is run it for 1000 epochs. The second part of the data is not seen by the neural network and is predicted as test set and accuracy is calculated from the confusion matrix. The accuracy from the confusion matrix is calculated as

$$
\text { Accuracy }=\frac{\text { No. of correct predictions }}{\text { Total no. of predictions }}
$$

Table 2 gives the accuracies of the train and test sets for only the Sum output and for three outputs present in the dataset before $\mathrm{K} 4$ cross validation.
Table 2: Train and Test set Accuracies of trained 4:2 ML Model

\begin{tabular}{|c|c|c|c|c|}
\hline \multirow{2}{*}{$\begin{array}{c}\text { Trained } \\
\text { ANN as } \\
\text { ML Model }\end{array}$} & \multicolumn{2}{|c|}{ For only } & \multicolumn{2}{c|}{ For three } \\
\cline { 2 - 5 } & $\begin{array}{c}\text { Sum output } \\
(\boldsymbol{\%})\end{array}$ & $\begin{array}{c}\text { outputs } \\
\text { Train set }\end{array}$ & $\begin{array}{c}\text { Test set } \\
\text { Train } \\
\text { set (\%) }\end{array}$ & $\begin{array}{c}\text { Test set } \\
(\boldsymbol{\%})\end{array}$ \\
\hline Exact [1-2] & 80 & 14.28 & 92 & 85.7 \\
\hline $\begin{array}{c}\text { Approximat } \\
\text { e [4] }\end{array}$ & 92 & 85.7 & 80 & 85.71 \\
\hline Proposed & 80 & 57.14 & 56 & 14.28 \\
\hline
\end{tabular}

The neural network becomes a complete validation model when the accuracy values obtained are equal or at least approximately equal. In Table 2, there is variation in these values. Also, when the model is executed for the second time, the trained accuracy value obtained is different from the first.

Thus, evaluation of this ML model has been done using a technique called $\mathrm{K} 4$ cross validation where the train set data is split into few folds (say ' $k$ '), then it is trained for 0 to k-1 folds and test it for last fold $\left(\mathrm{k}^{\mathrm{th}}\right)$. Such type of ' $\mathrm{k}$ ' folds are made for ' $p$ ' different combinations to train each combination for k-1 folds and the last one fold to test it. Later, average of these different accuracy values for different combinations are considered to compute mean and variance. The variance value obtained should be less indicating that the difference between trained accuracy values is less when calculated before and after evaluating K4 cross validation. These trained accuracy values obtained for trained and validated ML models are given in Table 3.

- The values given in brackets shows the absolute difference between the trained values before and after $\mathrm{K} 4$ cross validation which are to be less for perfect ML model.

- Though the accuracy values obtained after K4 cross validation are less for trained exact and proposed 4:2 compressor models, the difference in trained accuracy values before and after validation are also less which implies that the artificial neural network is behaving as 4:2 compressor.

- When only 'Sum' output is given in the dataset the trained accuracy value of the ML model behaving as proposed 4:2 compressor is less than [4] before and after $\mathrm{K} 4$ cross validation but the difference in these values is more for ML model behaving as [4] compressor.

- Similarly, when three outputs are present in the data set, the difference between the train sets is minimum for the ML model as proposed 4:2 compressor and maximum for exact ML model. 
Table 3: Train Set Accurcaies Before and After K4 Cross Validations

\begin{tabular}{|c|c|c|c|c|}
\hline \multirow{2}{*}{$\begin{array}{c}\text { Trained ANN } \\
\text { as }\end{array}$} & \multicolumn{2}{|c|}{ Train set Accuracy in \% } & \multicolumn{2}{c|}{ Train set Accuracy in \% } \\
\cline { 2 - 5 } ML & \multicolumn{2}{|c|}{ for only Sum output } & \multicolumn{2}{c|}{ for three outputs } \\
\cline { 2 - 5 } Model & before K4 & after K4 & before K4 & after K4 \\
\cline { 2 - 5 } & cross validation & cross validation & cross validation & cross validation \\
\hline Exact [1-2] & 80 & 73.91 & 92 & 69.57 \\
\hline $\begin{array}{c}\text { Approximate } \\
{[\mathbf{4}]}\end{array}$ & 92 & 100 & 80 & 60.87 \\
\hline Proposed & 80 & 78.26 & 56 & 65.22 \\
\hline
\end{tabular}

During the training and validation of a data set, the loss is also a measure which is used to interpret the performance of the model. The lower the loss, the trained ML model behaves better. The loss values of the three ML based compressor models for Sum only output and three outputs in the data sets before and after K4 cross validation for $1000^{\text {th }}$ epoch is shown in Table 4 and Fig. 3 and Fig. 4 displays the accuracy and loss values before and after cross validation.

Table 4: Train Set Accurcaies Before and After K4 Cross Validations

\begin{tabular}{|c|c|c|c|c|}
\hline \multirow{2}{*}{$\begin{array}{c}\text { Trained } \\
\text { ANN as }\end{array}$} & \multicolumn{2}{|c|}{$\begin{array}{c}\text { For Sum Outputs } \\
\text { only }\end{array}$} & \multicolumn{2}{c|}{ For three outputs } \\
\cline { 2 - 5 } & $\begin{array}{c}\text { Before } \\
\text { K4 }\end{array}$ & After K4 & $\begin{array}{c}\text { Before } \\
\text { K4 }\end{array}$ & $\begin{array}{c}\text { After } \\
\text { K4 }\end{array}$ \\
\hline $\begin{array}{c}\text { ML } \\
\text { Model }\end{array}$ & Loss & Loss & Loss & Loss \\
\hline Exact NN & 0.5287 & 0.46 & 1.0204 & 1.0047 \\
\hline $\begin{array}{c}\text { Approx } \\
\text { NN }\end{array}$ & 0.4815 & 0.756 & 0.687 & 0.7252 \\
\hline Prop NN & 0.4146 & 0.4612 & 0.679 & 0.3954 \\
\hline
\end{tabular}

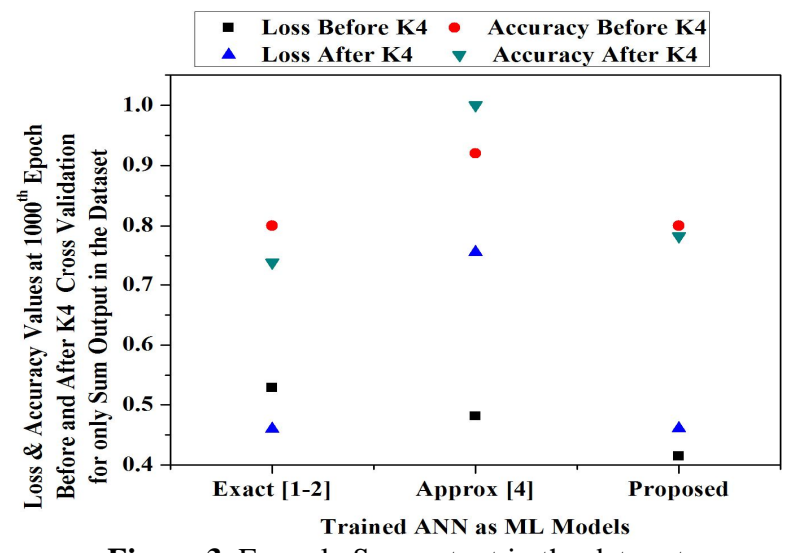

Figure 3: For only Sum output in the data set

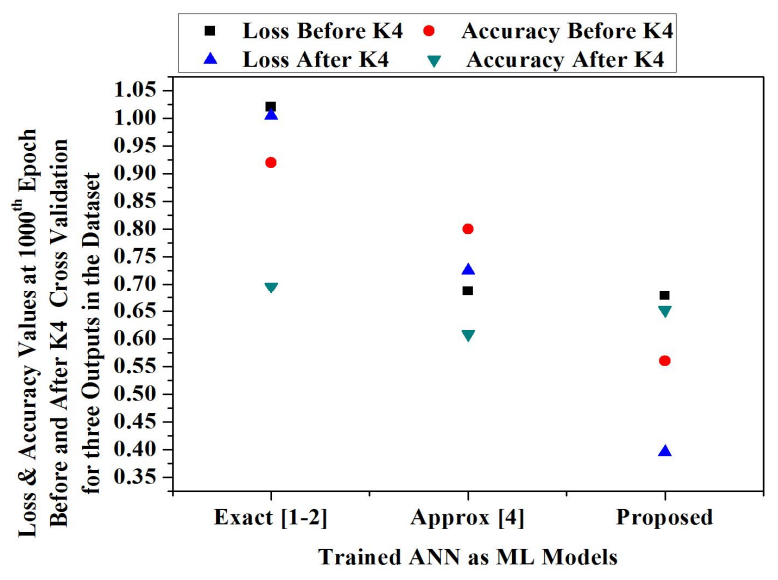

Figure 4: For three outputs: $\mathrm{C}_{\text {out }}$, Carry, Sum

This difference in trained and validated accuracy values for the above three ANN's before and after K4 cross validation is shown in Fig. 5

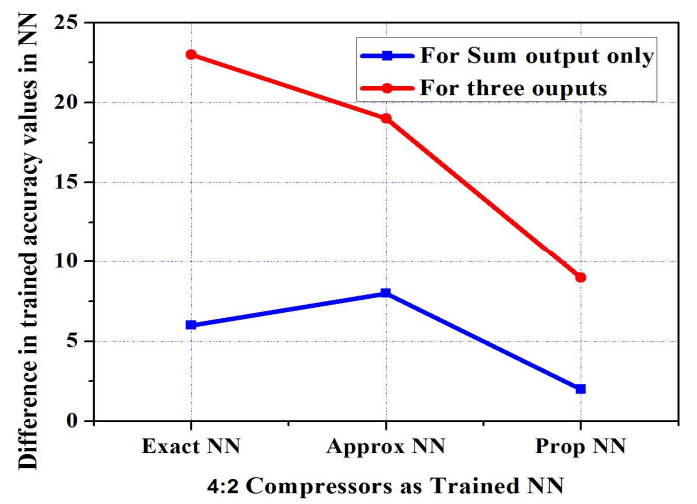

Figure 5: Difference in Trained Accuracy Values

After $\mathrm{K} 4$ cross validation the trade-off in bias and variance of the trained models is given in the form of variance and mean in Table 5. 
Table 5: Mean and Variance of ML Model after K4 Cross Validation

\begin{tabular}{|c|c|c|c|c|}
\hline \multirow{2}{*}{$\begin{array}{c}\text { Trained ANN } \\
\text { as }\end{array}$} & \multicolumn{3}{|c|}{$\begin{array}{c}\text { Trained ML model after k4 Cross } \\
\text { Validation }\end{array}$} \\
\cline { 2 - 5 } $\begin{array}{c}\text { ML } \\
\text { Model }\end{array}$ & \multicolumn{2}{|c|}{$\begin{array}{c}\text { For only Sum } \\
\text { output }\end{array}$} & \multicolumn{2}{c|}{ For three outputs } \\
\cline { 2 - 5 } & Mean & Variance & Mean & Variance \\
\hline Exact [1-2] & 0.3666 & 0.3055 & 0.5833 & 0.2813 \\
\hline $\begin{array}{c}\text { Approximate } \\
\text { [4] }\end{array}$ & 0.7833 & 0.2362 & 0.5166 & 0.2522 \\
\hline Proposed & 0.6 & 0.2808 & 0.3833 & 0.3077 \\
\hline
\end{tabular}

For the Proposed ML model, after validation, the variance and mean values are better for both the data sets. This indicates that the proposed ML model with only Sum output in the data set and ML model with three outputs in the data set has low bias and low variance, when compared with the exact and approximate [4] ML models.

\section{CONCLUSION}

With the intention to reduce the power consumption of a multiplier, approximations have been implemented on exact 4:2 compressors such that the proposed approximate 4:2 compressor has less error rate than the existing approximate compressors. The main purpose of applying classification ML algorithm is to classify the output of trained ML model as ' 1 or 0 ' such that the model functions as the proposed approximate 4:2 compressor. When the artificial neural network has been trained employing supervised ML algorithm, the performance of the proposed ML based approximate 4:2 compressor has been identified as the best since the difference in accuracy values between trained and validated ML models are less and has low bias and low variance. If the approximate techniques are employed, a compromise of accuracy in the architecture is expected but in this research work, the error rate is less and the trained ML model is also functioning as approximate 4:2 compressor with smaller loss values which indicates that it has been trained without flaws.

\section{ACKNOWLEDGEMENTS}

This research project was carried out at Center for Advanced Computing Research Laboratory (C-ACRL), Vardhaman College of Engineering. The authors would like to thank the management and faculty for their constant support throughout.

\section{REFERENCES}

[1] Veeramachaneni, S.,Krishna, K.M., Avinash,L., Puppala, S.R., and Srinivas, M.B., 2007 "Novel architectures for high-speed and low-power 3-2, 4-2 and 5-2 compressors,"
Proc. International Conference on VLSI Design (VLSID), pp. 324-329.

https://doi.org/10.1109/VLSID.2007.116

[2] Chang, C.H., Gu, J., and Zhang, M., "Ultra low voltage, low power CMOS 4-2 and 5-2 compressors for fast arithmetic circuits", IEEE Trans. Circuits Syst. I, Fundam. Theory Appl., 2004, 51, (10), pp. 1985-1997. https://doi.org/10.1109/TCSI.2004.835683

[3] Raphael Dornelle, Guilherme Paim, "A Power-Efficient 4-2 Adder Compressor Topology," 15th IEEE International conference on New Circuits and Systems Conference (NEWCAS), Strasbourg, France, 2017, pp. 281-284.

https://doi.org/10.1109/NEWCAS.2017.8010160

[4] Momeni, A., Han, J., Montuschi, P., and Lombardi, F., "Design and analysis of approximate compressors for multiplication," IEEE Transactions on Computers., 64 (4), 2015, pp. 984-994. https://doi.org/10.1109/TC.2014.2308214

[5] Darjn Esposito, Antonio Giuseppe Maria Strollo, Ettore Napoli, Davide De Caro, Nicola Petra, "Approximate Multipliers Based on New Approximate Compressors," IEEE Transactions on CAS-I: Regular papers, PP(99), 2018, pp. 1-14.

[6] Avinash Lingamneni, Christian Enz, Jean-Luc Nagel, Krishna Palem, and Christian Piguet, "Energy parsimonious circuit design through probabilistic pruning", Design, Automation and Test in Europe Conference and Exhibition, 2011. https://doi.org/10.1109/DATE.2011.5763130

[7] Liang, J Han, J Lombardi, F (2013), "New Metrics for the Reliability of Approximate and Probabilistic Adders," IEEE Transactions on Computers, 63(9), pp.1760-1771. https://doi.org/10.1109/TC.2012.146

[8]Zervakis, G et.al, "Design-Efficient Approximate Multiplication Circuits Through Partial Product Perforation," IEEE Trans. on VLSI Systems, 24(10), 2016, pp. 3105-3117. https://doi.org/10.1109/TVLSI.2016.2535398

[9] Peter, A, et.al, "Opportunities for Machine Learning in Electronic Design Automation," in ISCAS, 2018, Italy.

[10] Muhammad Shafique, Rehan Hafiz, et.al, "Adaptive and Energy- Efficient Architectures for Machine Learning: Challenges, Opportunities, and Research Roadmap", IEEE Computer Society Annual Symposium on VLSI (ISVLSI), 2017, Bochum, Germany. https://doi.org/10.1109/ISVLSI.2017.124

[11] Sashi Obilisetty, "Digital intelligence and chip design", International Symposium on VLSI Design, Automation and Test (VLSI-DAT), 2018, Taiwan. https://doi.org/10.1109/VLSI-DAT.2018.8373256

[12] Mounir Dhibi, Jitendra Pandey, Amine Al Kilani, "Majority Vote method for preferences detection: Application for Social Networks", International Journal of Advanced Trends in Computer Science and Engineering, Vol(8), No.(1), 2019. https://doi.org/10.30534/ijatcse/2019/08812019 